\title{
VEKTORANALYSIS
}

IN IHREN GRUNDZÜGEN

UND WICHTIGSTEN PHYSIKALISCHEN

ANWENDUNGEN

\author{
VON
}

\section{ARTHUR HAAS}

DR. PHIL., PROFESSUR FÜR PHYSIK AN DER UNIVERSITÄT WIEN

MIT 37 ABBILDUNGEN IM TEXT

ZWEITE, VERBESSERTE AUFLAGE

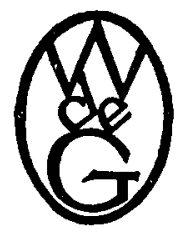

BERLIN UND LEIPZIG 1929

W A L T E R D E G R U Y T E R \& C O. VORMAIS $G$. J. GÖSCHEN'SCHE VERLAGSHANDLUNG . J. GUT IENTAG, VERLAGSBUCHHANDLUNG - GEORG REIMER - KARL J. TRUBNER - VEIT \& COMP. 
Copyright by Vereinigung wissenschaftlicher Verleger

Walter de Gruyter \& Co. in Leipzig, 1922

Manuldruds von F. Ullmann G. m. b. H., Zwickau Sa. 


\section{Aus dem Vorwort zur ersten Auflage.}

In den Vorlesungen, die ich im Wintersemester 1921/22 an der Wiener Universität hielt und die in diesem Buche wiedergegeben sind, sollte die Vektoranalysis nicht wie in anderen Büchern um ihrer selbst willen behandelt werden; es sollten vielmehr in diesen Vorlesungen die Grundlagen der Mechanik der Massenpunkte, der starren und deformierbaren Körper sowie die Grundlagen der MaxweLlschen Theorie und der Relativitätstheorie möglichst einfach mittels einer einheitlichen vektoriellen Methode entwickelt werden. $\mathrm{Zu}$ diesem Zwecke wurden in den Vorlesungen die Grundzüge der Vektor- und der Tensoranalysis dargestellt; doch wurde hierbei grundsätzlich niemals weiter gegangen, als es für die späteren physikalischen Anwendungen notwendig war.

In dem Buche wechseln rein mathematische Abschnitte mit solchen $a b$, in denen die gewonnenen mathematischen Erkenntnisse physikalisch verwertet werden. Eine scharfe Scheidung erschien mir notwendig, damit sich der Leser deutlich dessen bewußt werde, welche Zusammenhänge zwischen physikalischen Theoremen rein mathematischer Natur sind und welche nur unter Zuhilfenahme physikalischer Erfahrungstatsachen hergestellt werden können.

Von dem Hamizton schen Operator habe ich in diesem Buche nirgends Gebrauch gemacht, da ich glaube, dab seine Benutzung den Anfänger nur verwirrt; aus demselben Grunde erschien mir eine scharfe Betonung des Gegensatzes zwischen polaren und axialen Vektoren überflüssig. Auf eine Darstellung der verallgemeinerten, nichteuklidischen Tensoranalysis habe ich in diesem Buche verzichtet; ich glaubte dies um so eher tun zu sollen, als sich eine solche von mir verfaBte Darstellung ohnedies in meiner „Einführung in die theoretische Physik" (in dem Kapitel über allgemeine Relativitätstheorie) findet.

Wien, im Juni 1922.

\section{Arthur Haas.}

\section{Vorwort zur zweiten Auflage.}

Von der ersten Auflage unterscheidet sich die zweite außer durch mannigfache kleinere Verbesserungen in den anderen Abschnitten ror allem durch eine völlige Umarbeitung des § 13 (Spannung). Die Gliederung des Büchleins ist im übrigen unverändert geblieben.

Wien, im Juli 1929.

Arthur Haas. 
